\title{
Dihydroartemisinin Inhibits the Proliferation of Leukemia Cells K562 by Suppressing PKM2 and GLUTI Mediated Aerobic Glycolysis
}

This article was published in the following Dove Press journal:

Drug Design, Development and Therapy

Peng Gao ${ }^{1,2}$
Shuo Shen ${ }^{1,2}$
Xiaodong Li
Dandan Liu ${ }^{1,2}$
Yuqing Meng
Yanqing Liu ${ }^{1,2}$
Yongping Zhu
Junzhe Zhang
Piao Luo
Liwei Gu
'Institute of Chinese Materia Medica,
China Academy of Chinese Medical
Sciences, Beijing I00700, People's
Republic of China; ${ }^{2}$ Artemisinin Research
Center, China Academy of Chinese
Medical Sciences, Beijing I00700, People's
Republic of China; ${ }^{3}$ Institute of Chinese
Materia Medica, Gansu Provincial
Hospital of Traditional Chinese Medicine,
Lanzhou 730050, People's Republic of
China

Peng Gao',2

Shuo Shen ${ }^{1,2}$

Xiaodong $\mathrm{Li}^{3}$

Dandan Liu ${ }^{1,2}$

Yuqing Meng

Yanqing Liu ${ }^{1,2}$

Yongping $\mathrm{Zhu}^{1,2}$

Junzhe Zhang ${ }^{1,2}$

Piao Luo ${ }^{1,2}$

Liwei $\mathrm{Gu}^{1,2}$

'Institute of Chinese Materia Medica,

China Academy of Chinese Medical

Republic of China; ${ }^{2}$ Artemisinin Research

Center, China Academy of Chinese

Medical Sciences, Beijing 100700, People's

Materia Medica, Gansu Provincial

Lanzhou 730050, People's Republic of

Correspondence: Liwei Gu

Email Iwgu@icmm.ac.cn
Background: Leukemia threatens so many lives around the world. Dihydroartemisinin (DHA), as a typical derivative of artemisinin (ART), can efficiently inhibit leukemia, but the controversial mechanisms are still controversial. Many reports showed that tumor cells acquire energy through the glycolysis pathway, pyruvate kinase M2 (PKM2) plays a crucial role in regulating glycolysis. However, it is unclear whether PKM2 or other key molecules are involved in DHA induced cytotoxicity in leukemia cells. Thus, this paper systematically investigated the anticancer effect and mechanism of DHA on human chronic myeloid leukemia K562 cells.

Methods: In vitro, cytotoxicity was detected with CCK-8. Glucose uptake, lactate production and pyruvate kinase activity were investigated to evaluate the effect of DHA on K562 cells. To elucidate the cellular metabolism alterations induced by DHA, the extracellular acidification rate was assessed using Seahorse XF96 extracellular flux analyzer. Immunofluorescence, real-time PCR, and Western blotting were used to investigate the molecular mechanism.

Results: We found that DHA prevented cell proliferation in K562 cells through inhibiting aerobic glycolysis. Lactate product and glucose uptake were inhibited after DHA treatment. Results showed that DHA modulates glucose uptake through downregulating glucose transporter 1 (GLUT1) in both gene and protein levels. The cytotoxicity of DHA on K562 cells was significantly reversed by PKM2 agonist DASA-58. Pyruvate kinase activity was significantly reduced after DHA treatment, decreased expression of PKM2 was confirmed in situ.

Conclusion: The present study implicated that DHA inhibits leukemia cell proliferation by regulating glycolysis and metabolism, which mediated by downregulating PKM2 and GLUT1 expression. Our finding might enrich the artemisinins' antitumor mechanisms.

Keywords: tumor, leukemia, glycolysis, DHA, PKM2, GLUT1

\section{Introduction}

Cancer is a serious hazard to human health major refractory disease, and it is known that drug therapy has an important position in three major treatments of malignant tumors. According to the Global Cancer Report of the WHO Cancer Research Institute, there were about 18.1 million new cancer cases worldwide in 2018, which was a significant increase from the 14 million new cases in $2012 .{ }^{1}$ And the incidence and mortality of malignant tumor also had steadily increased. Hematological neoplasm malignancy accounts for approximately $3 \%$ of the total incidence of tumors. A widely 
divergent annual incidence estimate of chronic myeloid leukemia (CML) is about $0.4-1.0$ cases per 100,000 persons in the Asia-Pacific region. ${ }^{2}$ And there are more than 4 million cases of leukemia in China.

Compared to normal cells, cancer cells exhibit a unique characteristic through altered metabolic patterns. Cancer cells generally show increased glucose consumption ${ }^{3}$ and typically show high levels of glycolysis, even under aerobic conditions. ${ }^{4}$ This phenomenon is one of the hallmarks of cancer and known as "Warburg effect". 5,6 The glycolysis generates a large number of lactic acid, so-called metabolic waste. In turn, lactic acid would promote tumorigenesis. ${ }^{7}$ Hence, the special dependence of cancer cells on glycolysis makes them vulnerable to the specific glycolysis target inhibitors. Acting as a d-glucose mimic, 2-Deoxy-D-glucose (2DG) has been reported as a glycolysis inhibitor. Because of the formation and intracellular accumulation of 2-deoxy-d-glucose-6-phosphate (2-DG6P), 2DG could inhibit the function of glucose6-phosphate isomerase and hexokinase, ${ }^{8}$ which is the entry-point enzyme for glycolysis. ${ }^{9}$ Therefore, 2DG has been studied as a prospective therapeutic compound that targets metabolic alterations of tumor cells. ${ }^{10}$ Pyruvate kinase M2 isoform (PKM2), which is specifically expressed in rapidly proliferating cells such as cancer cells. PKM2 is the rate-limiting enzyme and catalyzes the last step of glycolysis. ${ }^{11}$ The high expression and activity of PKM2 are essential for the "Warburg effect". ${ }^{12}$ Based on the pivotal role, PKM2 and associated signaling pathways may be the target of improving the anticancer therapeutic strategies in future. ${ }^{13}$ Glucose transporter 1 (GLUT1), is also known as solute carrier family 2 (SLC2A1), which is used to mediate transmembrane transport of glucose into cells. ${ }^{14}$ GLUT1 is the most important member of the mammalian facilitative glucose transporter family, which is the main limit of cell glucose metabolism and excessively expressed in almost all kinds of tumors. ${ }^{15,16}$ Recently, GLUT1 has become the new targets of antitumor therapy. ${ }^{17}$

Artemisinin was a sesquiterpene lactone compound with an endoperoxide bridge structure, which was isolated from the leaves of Artemisia annua L. by Chinese scientist Tu Youyou in the 1970s. ${ }^{18}$ Dihydroartemisinin (DHA) is a typical derivative of artemisinin, which is reported as the active metabolite of artemisinin and its derivatives (ARTs). In addition to their antimalarial effect, ${ }^{19}$ ARTs have good antitumor activity. ${ }^{20}$ The antitumor mechanism of artemisinin derivatives is still unclear now, and the possible mechanisms include oxidative stress response involving iron, $^{21}$ ferroptosis and cell cycle arrest, ${ }^{22}$ apoptosis $^{23}$ and autophagy induction, ${ }^{24}$ anti-angiogenesis, ${ }^{25}$ and invasion and metastasis inhibition. ${ }^{26}$ However, the relationship between artemisinin's derivatives and energy metabolism in cancer has rarely been reported clearly, especially its effect on aerobic glycolysis.

In the present study, DHA was selected as a representative compound to investigate the effect of artemisinin's derivatives on "Warburg effect" in chronic myelogenous leukemia K562 cells. Aimed to observe the correlation between DHA and aerobic glycolysis in vitro, as well as explore the exact effects of DHA on proliferation and energy metabolism in leukemia cells.

\section{Materials and Methods Materials and Cell Line}

Human chronic myeloid leukemia cells K562 and hepatoma carcinoma cells HepG2 were purchased from the Institute of Basic Medical Sciences of Chinese Academy of Medical Sciences (Beijing, China). DHA was purchased from Chongqing Huali Wulingshan Medicine Co., Ltd. (Lot No. C00220160402). 2DG (Cat. No. D8930) and Hoechst33342 (Cat. No. B8040) were purchased from Solarbio Life Sciences (Beijing, China). DASA-58 was purchased from MedChemExpress LLC (Cat. No. HY19330/CS-5257, NJ, USA). RPMI 1640 medium and penicillin-streptomycin solution were purchased from Hyclon, fetal bovine serum (FBS) was purchased from GIBCO (Grand Island, NY, USA). The primary antibodies used were as follows: Antibodies specific for Human GLUT1 (\#MAB14181, R\&D), P53 (\#NBP2-34495, Novusbio), c-Myc (\#NBP2-45144, Novusbio), $\beta$-actin (\#8224, Abcam), PKM2 (\#60268-1-lg, Proteintech) were used. CELL-TAK $^{\mathrm{TM}}$ was purchased from Corning (Cat. No. 354240, NY, USA). DASA-58 was purchased from MedChemExpress LLC (Lot\#42425). DMSO and other chemical reagents were purchased from Sigma (St. Louis, USA).

\section{Cytotoxicity Analysis}

The cytotoxicity of DHA was confirmed on K562 cells using the CCK- 8 method. The cells were seeded in 96-well culture plates at a density of 5000 cells/well. Then, the cells were treated with DHA at concentrations ranging from $1.28 \mathrm{nM}$ to $100 \mu \mathrm{M}$ at $37^{\circ} \mathrm{C}$ for 24,48 and $72 \mathrm{hrs}$. After that, the drug solution was added with CCK-8 
(DOJINDO, Japan), and co-incubated with cells for another $2 \mathrm{hrs}$. The absorbance at $450 \mathrm{~nm}$ was recorded using a microplate reader (Molecular Devices, SpectraMax Plus 384). The TGI (tumor cell growth inhibition ratio) was calculated according to the following formula:

$$
T G I(\%)=\frac{C-T}{C} \times 100 \%
$$

$\mathrm{T}$ represented the average absorbance value of treated groups, and $\mathrm{C}$ represented the average absorbance value of the control group. Here, the activation of PKM2 was used DASA-58, a well-characterized small molecule.

\section{Lactate Production Assays}

Cells were seeded onto 24-well plates at a density of $2 \times 10^{5}$ cells per well. Then, cells were treated with DHA at concentrations ranging from $160 \mathrm{nM}$ to $100 \mu \mathrm{M}$ for 24 and $48 \mathrm{hrs}$. The culture supernate was taken after the cells were centrifuged. Subsequently, cells' pellets were resuspended in $500 \mu \mathrm{L}$ medium and then lysed by ultrasonic ( 50 $\mathrm{W}$, ultrasonic $2 \mathrm{~s}$, interval $3 \mathrm{~s}$ for 5 times). Then, the lactate concentrations in cell lysates were detected using the Lactate Assay Kit (K627-100, BioVision, Milpitas, USA) according to the manufacturer's instructions; then, centrifuged at $4^{\circ} \mathrm{C}$ at $12,000 \mathrm{RPM}$ for $5 \mathrm{mins}$, and the supernatant was taken for analysis. The absorbance at 450 $\mathrm{nm}$ was recorded using a microplate reader (Molecular Devices, SpectraMax Plus 384).

\section{Glucose Uptake Assays}

Glucose uptake assays were detected by a Colorimetric Assay Kit (K676-100, BioVision, Milpitas, USA). K562 cells were plated at a density of 1500 per well in 96-well plates and cultured with DHA at different concentrations for $96 \mathrm{~h}$ and washed with PBS and continued to culture in glucose-free RPMI 1640 medium (Gibco, USA) for overnight. The cells were washed with PBS and added $100 \mu \mathrm{L}$ Krebs-Ringer- Phosphate-HEPES (KRPH) buffer containing 2\% BSA (KRPH buffer: $20 \mathrm{mM}$ HEPES, $5 \mathrm{mM} \mathrm{\textrm {KH } _ { 2 }}$ $\mathrm{PO}_{4}, 1 \mathrm{mM} \mathrm{MgSO}$, $1 \mathrm{mM} \mathrm{CaCl} 2,136 \mathrm{mM} \mathrm{NaCl}, 4.7 \mathrm{mM}$ $\mathrm{KCl}, \mathrm{pH}$ 7.4). Then, the culture media were added with 10 $\mu \mathrm{M}$ 2-Deoxy-2-[(7-nitro-2,1,3-benzoxadiazol- 4-yl) amino]-D-glucose (2-DG) and cells were cultured for an additional $20 \mathrm{~min}$. Measure the absorbance at $412 \mathrm{~nm}$ in a microplate reader (Molecular Devices, SpectraMax I3X) at $37^{\circ} \mathrm{C}$ every 5 mins until the 100 pmol standard reaches 1.5-2.0 OD.

\section{Pyruvate Kinase Activity}

Pyruvate kinase activity was detected using a commercial kit (Solarbio BC0540, Beijing, China). All measurements were performed following the manufacturer's instructions. Briefly, cells were collected into an EP tube, and the supernatant was discarded after centrifugation. Extract was added according to the number of cells (about $5 \times 10^{6}$ cell: $1 \mathrm{~mL}$ ). The cells were ultrasonically broken on ice (200 W, ultrasonic $3 \mathrm{~s}$, interval $10 \mathrm{~s}$, repeat for 30 times); then held on $95^{\circ} \mathrm{C}$ water bath for 10 mins, and after cooling to room temperature, the solutions were centrifuged $8000 \mathrm{~g}$ at $25^{\circ} \mathrm{C}$ for $10 \mathrm{mins}$. Then, these supernatants were taken for analysis of glucose levels, samples were detected according to the User Guide and the absorbance value was tested at $340 \mathrm{~nm}$ using UV-spectrophotometer.

\section{Extracellular Acidification Rate (ECAR) Measurement}

The XF96 extracellular flux analyzer (Seahorse Bioscience, Billerica, USA) was used to detect real-time changes in the extracellular acidification rate (ECAR). Briefly, the culture plate was pre-coated CELL-TAK, and K562 cells were seeded at a density of $3 \times 10^{4}$ cells per well and cultured overnight. Cells were treated with different concentrations of DHA for $12 \mathrm{~h}$ prior to the measurement. Then, the cells were washed once. A solution of $1 \mathrm{mM}$ glutamine (Seahorse XF, Cat No. 103579-100, USA) was added in XF RPMI base medium. For ECAR analysis (Glycolysis Stress Test), $10 \mathrm{mM}$ glucose, $1 \mu \mathrm{M}$ oligomycin and $50 \mathrm{mM} 2-\mathrm{DG}$ (Agilent, Seahorse XF Test Kits, Cat No.103020-100, USA) were added. All measurements were performed following the manufacturer's instructions and normalized with the number of cells counted with Hoechst33342 (Solarbio, Beijing, China) in each well at the end of the seahorse experiments.

\section{RNA Preparation and Florescent Real-Time PCR}

K562 cells were plated at a density of $4 \times 10^{5} /$ well in 6 -well plates and cultured with or without DHA. After treatment with DHA for $24 \mathrm{~h}$ or $48 \mathrm{~h}$, total RNA was isolated from cultured cells using RNA extraction kit (DP431, TIANGEN BIOTECH, Beijing, China) and the concentration of total RNA was measured spectrophotometer (DS-11, Denovix, US). cDNA was synthesized using a FastKing RT Kit (with gDNase) (KR116, TIANGEN BIOTECH, Beijing, China). RTPCR was performed using the $\operatorname{TransStart}^{\circledR}$ Green qPCR 
SuperMix UDG kit (AQ111-01, TRANSGEN BIOTECH, Beijing, China) according to the manufacturer's protocol. Quantitative RT-PCR was performed on a qTOWER3.0 PCR instrument (Jena, Germany) using two-stage program parameters provided by the manufacturer as follows: 2 mins at $50^{\circ} \mathrm{C}, 10 \mathrm{mins}$ at $95^{\circ} \mathrm{C}$, and then 40 cycles of $95^{\circ} \mathrm{C}$ for $15 \mathrm{~s}$, $60^{\circ} \mathrm{C}$ for $32 \mathrm{~s}$. Each sample was tested in triplicate using quantitative RT-PCR, and samples obtained from three independent experiments were used for the analysis of relative gene expression data using the $2^{-\Delta \Delta \mathrm{CT}}$ method. The primers were used in this study were listed in Supplementary Table S1. The quantity of each transcript was calculated as described in the instrument manual and normalized to the amount of GAPDH, a housekeeping gene. Where the $\Delta \Delta \mathrm{Ct}=$ $\left[\left(\mathrm{Ct}_{\text {target gene }}-\mathrm{Ct}_{\text {GAPDH }}\right)_{\text {Treatment group }}-\left(\mathrm{Ct}_{\text {target gen }} \mathrm{e}-\right.\right.$ $\left.\left.\mathrm{Ct}_{\text {GAPDH }}\right)_{\text {Control group }}\right]$.

\section{Western Blot Assays}

K562 cells were seeded at a density of $4 \times 10^{5} /$ well in 6-well plates. After treatment, cells were lysed with lysis buffer (10 $\mu \mathrm{L}$ PMSF, $100 \mathrm{mM}$ was added to $1 \mathrm{~mL}$ RIPA, Solarbio, Beijing, China) on ice for 30 mins. Cells lysates were separated with SDS-PAGE and blotted on a PVDF membrane, which were blocked with 5\% skim milk in PBS containing $0.1 \%$ Tween 20 (TBST). Then, the PVDF membrane was incubated overnight with the primary antibodies at $4^{\circ} \mathrm{C}$. The bound antibodies were detected by secondary HRP-conjugated antibodies and the bands were visualized using the ECL system (Thermo Fisher Scientific, USA). Bands images were obtained by using the Proteinsimple Digital imaging system (Flour Chem R, USA).

\section{Immunostaining Analysis and Protein Measurement in situ}

The cells were seeded on glass coverslips $(12 \mathrm{~mm})$ which coating with lysine and cultured in or absence $4 \mu \mathrm{M}$ DHA for 24 hrs. After treatment, cells were fixed in 4\% paraformaldehyde for 15 mins at room temperature. After fixation, cells were permeabilised with a wash buffer $(1 \times$ PBS containing $0.2 \%$ Triton-X 100). Afterwards, the coverslips were incubated with a blocking buffer (wash buffer with 5\% skim milk) for $1 \mathrm{hr}$ at room temperature and subsequently incubated with a mouse monoclonal antibody specific to PKM2 in a 1:200 dilution overnight at $4^{\circ} \mathrm{C}$. Following three washes in TBST, the coverslips were incubated with Alexa Fluor 647 conjugated secondary antibodies (Molecular probes containing $2 \mu \mathrm{g} / \mathrm{mL}$ Hoechst33342) for $1 \mathrm{hr}$ at room temperature protected from light. Free secondary antibodies were removed by three washes in TBST, and the coverslips mounted on glass slides were detected with a laser scanning confocal microscope (SP8, Lecia, Germany).

\section{Statistical Analysis}

All group results were expressed as the mean \pm standard deviation of at least three repeated samples. Differences between groups were analyzed using Graphpad Prism and SPSS statistical software (SPSS, Chicago, IL). It was regarded as statistically significant when $\mathrm{P}<0.05$.

\section{Results}

\section{DHA Inhibits K562 Cells Proliferation}

Cell viability assay showed that DHA could inhibit the growth of K562 cells in a dose and time-dependent manner (Figure 1A-C). After 24-72 hrs exposed to K562, the $\mathrm{IC}_{50}$ values of DHA were about $1.1-2.6 \mu \mathrm{M}$. The cytotoxicity of DHA on leukemia cells was significantly stronger than that on other cancer cells (data not shown here). When PKM2 agonist DASA-58 was added, cytotoxicity of DHA was partly reversed (Figure 1D). It is suggested that the cytotoxicity of DHA partly acted through PKM2.

\section{DHA Regulated Glycolysis Metabolism}

Lactic acid is produced as a by-product of aerobic glycolysis, but not aerobic respiration. Therefore, the yield of lactic acid can be used to evaluate the level of glycolysis activity. ${ }^{27}$ To evaluate whether the glycolytic activity was suppressed following DHA administration, lactate production and glucose uptake were detected. The results demonstrated that DHA significantly inhibited lactic acid production in cells when compared with controls $(\mathrm{P}<0.05$; Figure $2 \mathrm{~A}$ and $\mathrm{B})$. In addition, glucose uptake was also reduced upon DHA treatment. Meanwhile, DHA significantly reduced cellular pyruvate kinase activity $(\mathrm{P}<0.05$; Figure $2 \mathrm{C}$ and $\mathrm{D})$. The above results showed that DHA regulates glycolysis metabolism.

\section{DHA Suppresses Aerobic Glycolysis in K562 Cells}

To further elucidate the detailed cellular metabolism alterations induced by DHA, the extracellular acidification rate (ECAR) was assessed using Seahorse XF96 extracellular flux analyzer. As shown in Figure 3, ECAR parameters including the glycolytic activity and capacity decreased in a concentrationdependent manner in K562 cells following DHA 

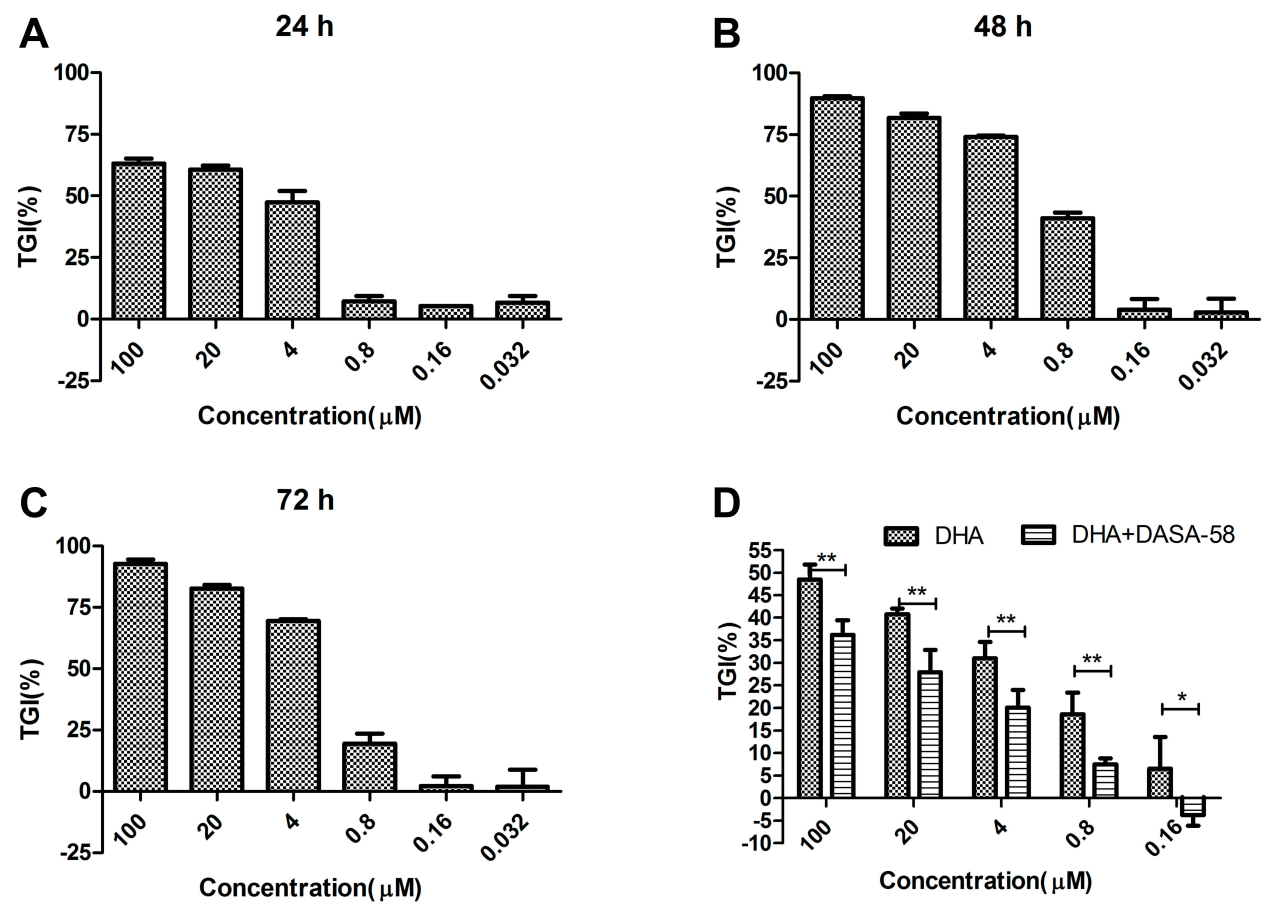

Figure I Cytotoxicity of DHA on K562 at (A) 24 hrs, (B) 48 hrs and (C) 72 hrs. (D) The growth inhibition of DHA after 24 hrs together with $40 \mu M$ DASA-58 (a PKM2 agonist). Tumor cell growth inhibition was determined by CCK-8 after treated with various concentrations of DHA at different times. All data were obtained from three independent experiments $(n=4)$ and all values are represented as mean $\pm S D(* P<0.05, * * P<0.01)$.

administration compared with the control group (Figure 3). Altogether, these results clearly indicated that DHA could suppress aerobic glycolysis in leukemia cells in vitro.

\section{DHA Regulated mRNA Expression of Glycolysis Related Gene}

To verify the mechanism of the inhibition effect of DHA on glycolysis, we tested the expression of $c-M y c, G L U T 1$, $P K M 2$ and other regulated genes after DHA treatment with different concentrations $(0,0.8$, and $4 \mu \mathrm{M})$ and 2DG (10 $\mathrm{mM})$. The results showed that DHA treatment downregulated the relative expression of GLUT1, PKM2, LDH-A and $c-M y c$ (Figure 4). The relative changes of PDK1, P53, HIF$1 \alpha$, HK2, and PFK1 expression were modest, with most genes being altered by less than 2-fold (Supplementary Figure S1). These results suggested that DHA suppressed aerobic glycolysis through inhibiting PKM2 expression, which leads to inhibition of cell proliferation.

\section{DHA Regulated Protein Expression Involved in Glycolysis}

To further explore the underlying mechanism, we investigated a series of relative key proteins involved in cell energy metabolism, including c-Myc, p53, GLUT1 and PKM2. DHA significantly decreased the level of GLUT1 and PKM2 expression (Figure 5A-C), and the effect of c-Myc and p53 was less pronounced (data not shown). The expression of PKM2 was gradually decreased by DHA. K562 and human liver cancer HepG2 cells (adherent cells) were used as models (Figure 5D and E). After incubation with $4 \mu \mathrm{M}$ DHA for $24 \mathrm{hrs}$, cells were stained with Hoechest33342 and PKM2 fluorescent antibodies, respectively. Detection of PKM2 expression in situ was performed. The expression of PKM2 was markedly decreased in the whole cell. The above results demonstrated that DHA regulates protein expression which involved in glycolysis.

\section{Discussion}

It is known that the rapid growth of cancer cells needs large amounts of glucose and furious increases in glucose metabolite. The "Warburg effect" can be reflected in a general elevation of metabolic rates to support increased proliferation, as well as a shift in preferred energy source from occurs in oxidative phosphorylation to aerobic glycolysis. ${ }^{5}$ Besides tumor cells, other highly proliferating cells also exhibit "Warburg effect", such as activated M1 


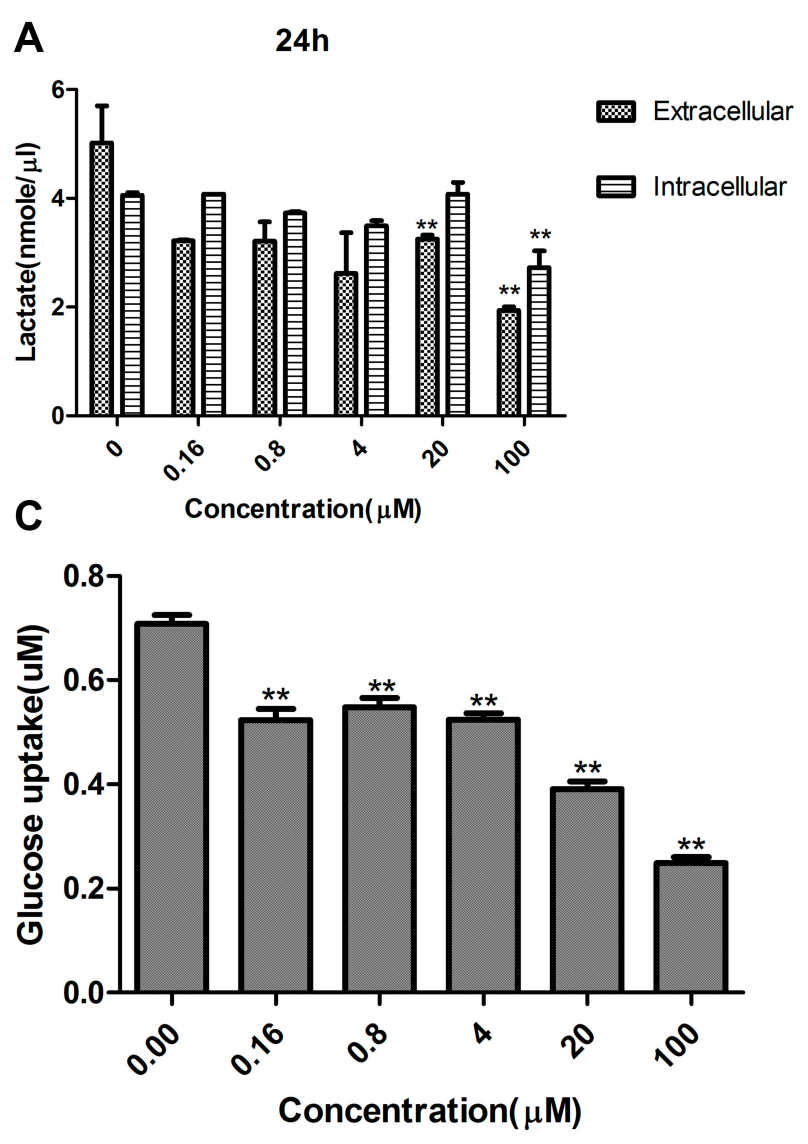

B
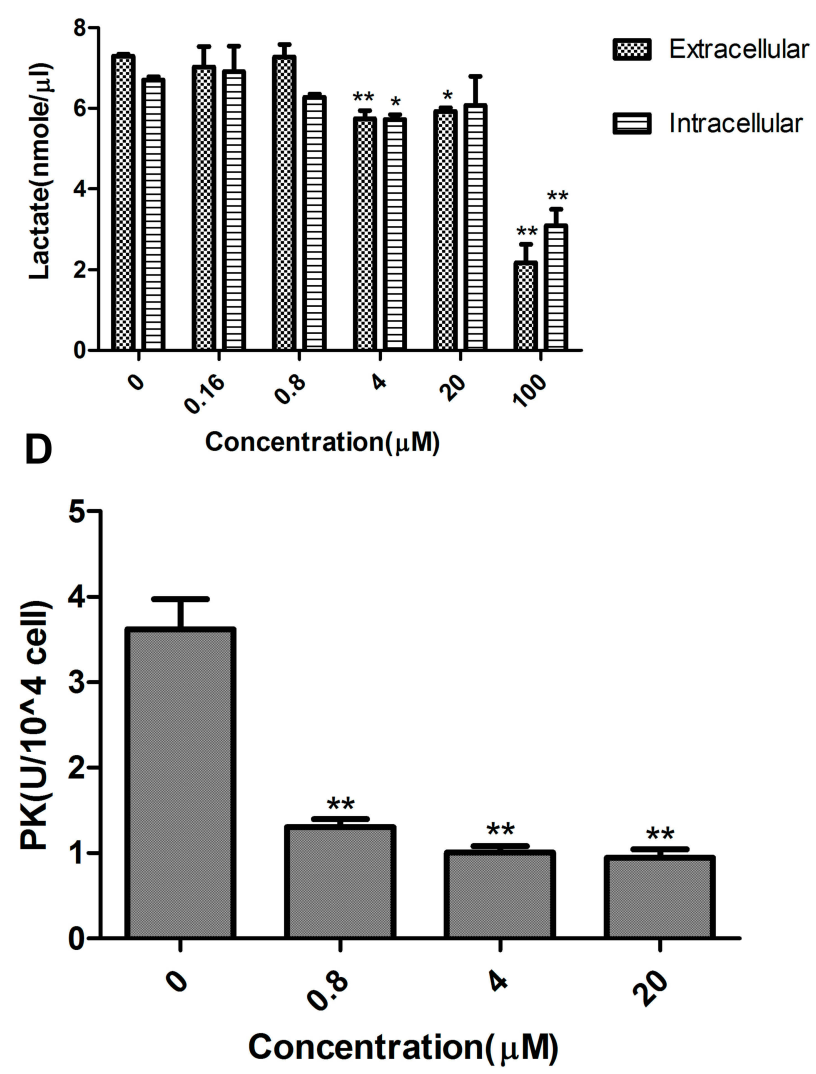

Figure 2 Effect of DHA on key metabolites secretion. (A, B) Effect of DHA on lactic acid secretion. DHA can dose-dependent inhibit the secretion of lactic acid. After 24 $48 \mathrm{hrs}$ of treatment, both intracellular and extracellular lactate levels of $\mathrm{K} 562$ cells were significantly inhibited. (C) Glucose uptake was suppressed by DHA. After the treatment of DHA at different concentrations $(0.16 \mu \mathrm{M}$ to $100 \mu \mathrm{M})$ for 96 hrs, the level of Glucose was determined. (D) After the treatment of DHA at different concentrations $(0.8 \mu \mathrm{M}$ to $20 \mu \mathrm{M})$ for $24 \mathrm{hrs}$, pyruvate kinase activity was determined in $\mathrm{K} 562$ cells. All data were obtained from three independent experiments ( $\mathrm{n}=3$ ) and all values are represented as mean $\pm S D(* P<0.05, * * P<0.01)$.

macrophages and Th17 cells. This means that there are not only cancer cells with the "Warburg effect" but also other diseases such as diabetes, sepsis, autoimmune diseases, and atherosclerosis. ${ }^{28}$

The high glycolysis rate in tumor cells is due to the upregulation of enzymes in glycolysis, for example, hexokinase 2 (HK2), 6-phosphofructo-1-kinase (PFK1), pyruvate kinase (PK), and so on. ${ }^{29} \mathrm{HK} 2$ is the isoform expressed in cancer cells, which regulates the first step in glycolysis. ${ }^{30}$ It was regulated by p53 and HIF-1 and reported as the target of $2 \mathrm{DG} .{ }^{8}$ Another important kinase in glycolysis is pyruvate dehydrogenase kinase (PDK). PDK regulates the uptake of cytosolic pyruvate into the TCA cycle. It is reported that when PDK was inhibited, the mitochondrial respiration was increased and aerobic glycolysis was reduced. ${ }^{31}$ The conversion of glucose, pyruvate and glutamine to lactate involves the enzyme lactate dehydrogenase (LDH). LDH type A (LDH-A) is induced by c-MYC, which is considered the key metabolic players. ${ }^{32}$ The crucial kinase and molecules were analyzed by RT-PCR in this study. Our results showed the relative expression of GLUT1, PKM2, LDH-A and $c-M y c$ was significantly downregulated by DHA (Figure 4 and Supplementary Figure S1).

Pyruvate kinase (PK) is a glycolytic enzyme that catalyzes a reaction generating pyruvate and ATP from phosphoenolpyruvate (PEP) and ADP. ${ }^{33}$ PKM1 and PKM2 are encoded by the $P K M$ gene. PKM1 is expressed in many adult tissues, such as muscle and brain. In contrast to its splice variant, PKM2 is expressed in rapidly proliferative cells such as cancer cells, embryonic cells and adults stem cells. It is reported that the original tissue-specific PK is replaced by PKM2 during tumorigenesis. ${ }^{34}$ Considering the key enzyme in regulating glycolysis and oxidative phosphorylation in tumor, ${ }^{35}$ PKM2 could be a potential target for cancer treatment. ${ }^{36}$ Recently, PKM2 was found to play an important role in many other diseases, for example, heart failure ${ }^{37}$ and osteoarthritis. ${ }^{38}$ Including 

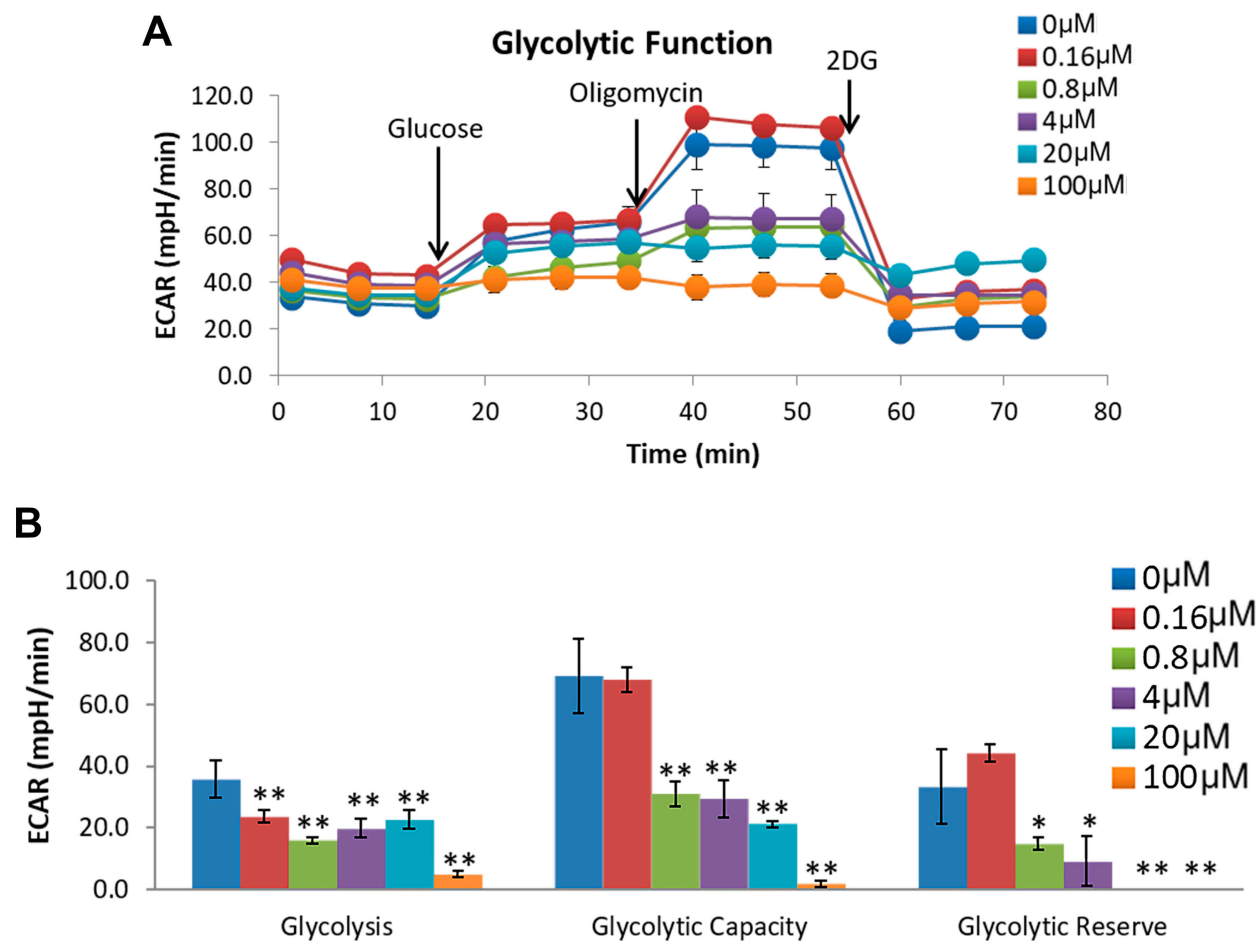

Figure 3 DHA suppressed aerobic glycolysis. (A, B) K562 cells were treated with different concentrations of DHA $(0.16-100 \mu M)$ for 12 h. The extracellular acidification rate (ECAR) was detected by the Seahorse XF96 extracellular flux analyzer using the Glycolysis Stress Test Kit. As expected, DHA treatment decreased ECAR in K562 leukemia cells. All data were obtained from twice independent experiments $(n=4)$ and all values are represented as mean $\pm S D(* P<0.05$, $* * P<0.0 \mathrm{I})$.
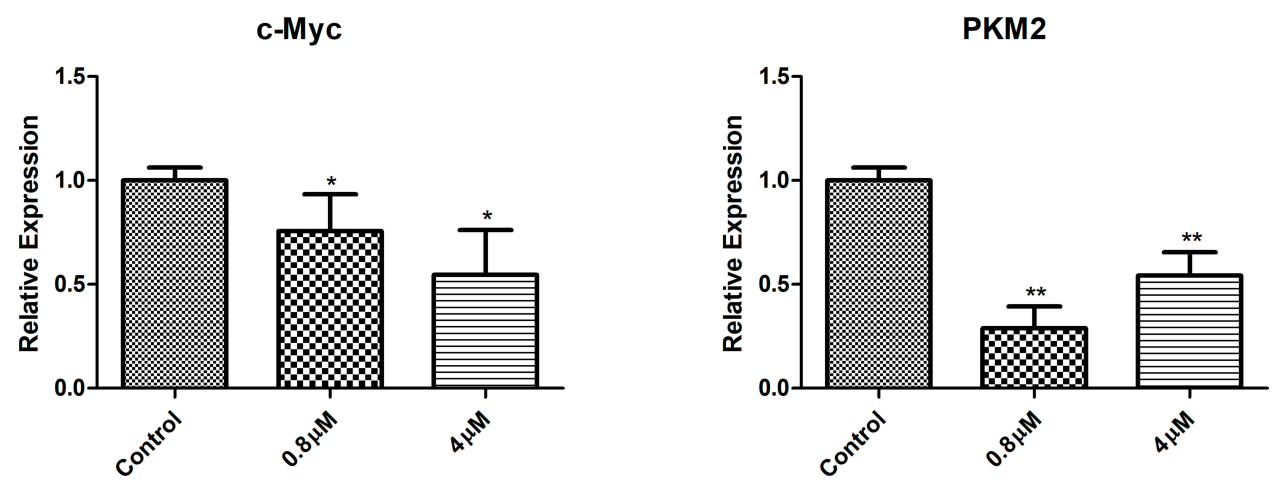

\section{LDH-A}
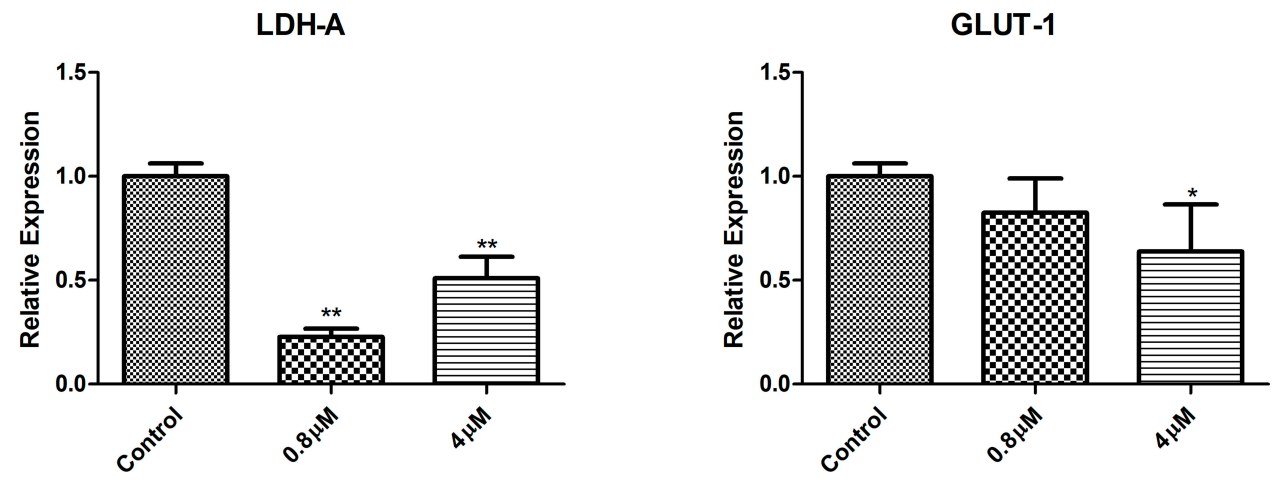

Figure 4 Influence of DHA on the aerobic glycolysis related mRNA expression level. All data were obtained from three independent experiments ( $\mathrm{n}=3$ ) and all values are represented as mean $\pm S D(* P<0.05, * * P<0.01)$. 

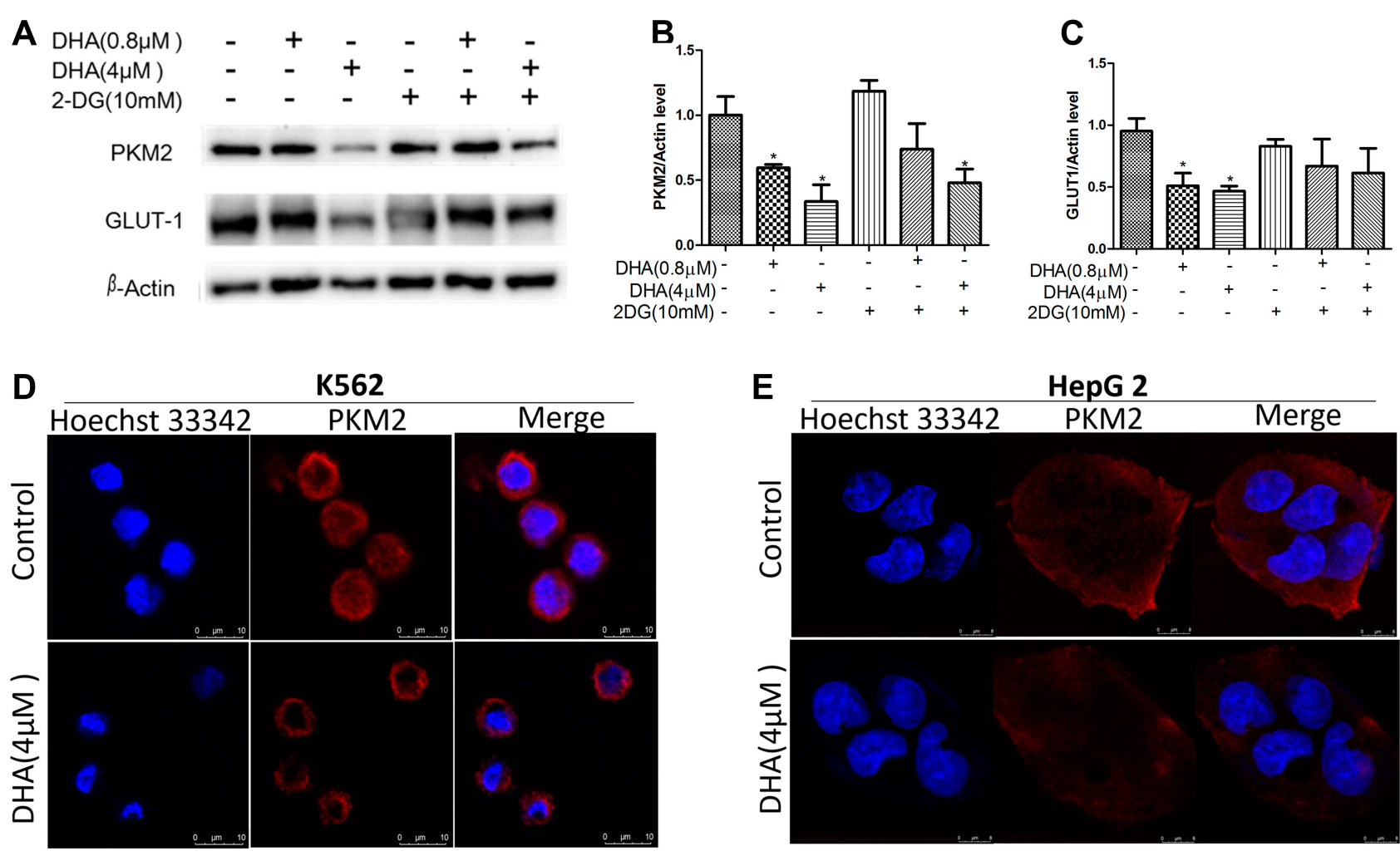

Figure 5 DHA suppresses PKM2 and GLUTI expression. (A) K562 cells were treated with different concentrations of DHA (0.8 $\mu$ M and $4 \mu M)$ for 24 h. The protein expression level was detected by the western bolt assay. PKM2 (B) and GLUTI (C) expression levels were reduced. Meanwhile, 2DG does not interfere with the efficacy of DHA. Immunofluorescence assay indicated that DHA effectively suppressed the expression and nuclear transportation of PKM2 both in (D) K562 and (E) HepG2 cells. Scale bars indicate $10 \mu \mathrm{m}$. All data were obtained from three independent experiments $(n=3)$ and all values are represented as mean \pm SD $(* P<0.05)$.

our research, many reports found that ARTs exhibit more cytotoxicity in leukemia than other tumor cells. ${ }^{39,40}$ Here, we indicated that DHA inhibited lactic acid secretion (Figure 2) and glycolysis in chronic myeloid leukemia cells (Figure 3). Furthermore, the present study shows that DHA suppressed the expression of PKM2 either in gene or in protein level (Figures 4 and 5). To confirm our conclusions, we also proved PKM2 expression in situ with adherent cell HepG2, besides K562 cells. Consistent with our results, a study on esophageal cancer also identified DHA inhibits glycolysis by downregulating PKM2. ${ }^{41}$

It is often observed glucose transporters (GLUTs) overexpression to adjust the "Warburg effect" in cancer cells. As mentioned, it is now widely accepted that GLUT1 provide promising targets for anti-cancer drugs. ${ }^{15}$ In our study, we also found that the expression of GLUT1 was gradually suppressed in both gene and protein levels by DHA. It suggests that DHA could block the uptake of glucose by suppressing GLUT1 expression in K562 cells. Taken together, GLUT1 may serve as a new target for leukemia. DHA regulates the metabolism events through inhibiting both GLUT1 and PKM2.
Many studies have reported that as a glucose analogue, 2DG could inhibit glycolysis through inhibiting hexokinase and play a direct or auxiliary role in antitumor. ${ }^{10,28,42}$ Confusingly, the combination of 2DG and DHA had neither synergy nor antagonism on the expression of PKM2 and GLUT1 in our study (Figure 5). The potential mechanisms of DHA and 2DG on glycolysis might be different. Further research is needed to investigate the specific mechanism.

In conclusion, our present study demonstrated that DHA inhibited cell proliferation in human chronic myeloid leukemia cells through regulating PKM2 and GLUT1 mediated aerobic glycolysis. The putative mechanism of DHA interacting with tumor cells is showed in Figure 6. DHA inhibited glycolysis, which in turn influenced the cell energy metabolism. Artemisinin's derivatives might become a prospective strategy act against cancer and other diseases.

\section{Acknowledgments}

Human chronic myeloid leukemia cells K562 were provided by Doctor Qi Li who purchased cells from the 


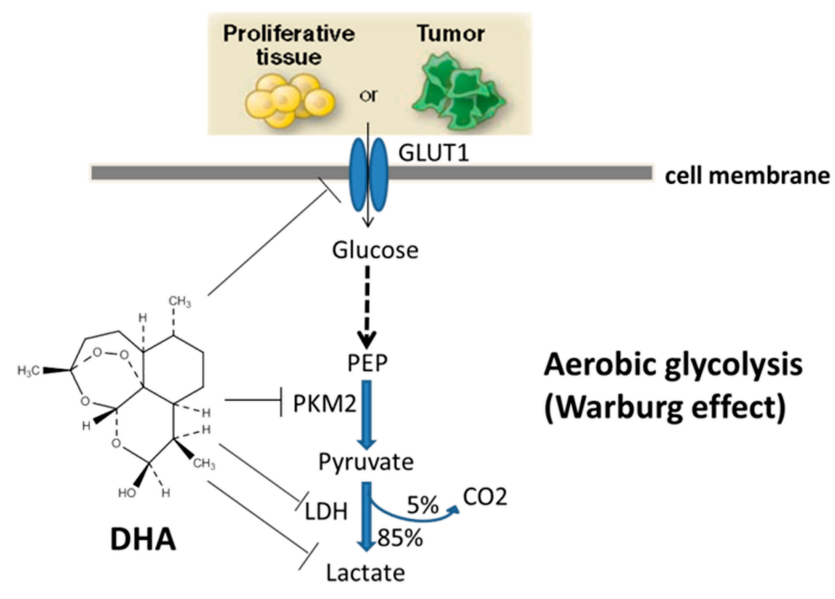

Figure 6 Summary of the mechanisms of cytotoxicity of human leukemia cell line K562 induced by DHA. Our results showed that DHA decreased cell proliferation in human chronic myeloid leukemia cells through inhibiting glycolysis. DHA could inhibit lactic acid secretion and repress glucose uptake by inhibiting GLUTI. PKM2 was found to play an important role in "Warburg effect". Our results indicated that DHA effectively suppressed the expression of PKM2.

Institute of Basic Medical Sciences of Chinese Academy of Medical Sciences. We thank Professor Lan Yang for supplying DHA and Doctor Jianying Shen for the early stages of project discussions. This work was supported by a grant from the National Natural Science Foundation of China (Grant No. 81603346) and the Fundamental Research Funds for the Central public welfare research institutes (Grant No. ZZ13-YQ-103).

\section{Disclosure}

The authors declare no conflict of interest.

\section{References}

1. Bray F, Ferlay J, Soerjomataram I, Siegel RL, Torre LA, Jemal A. Global cancer statistics 2018: GLOBOCAN estimates of incidence and mortality worldwide for 36 cancers in 185 countries. CA Cancer J Clin. 2018;68(6):394-424. doi:10.3322/caac.21492

2. Chang CS, Lee K, Yang Y-H, Lin M-T, Hsu C-N. Estimation of CML incidence: disagreement between national cancer registry and health claims data system in Taiwan. Leuk Res. 2011;35(5):e53-e54. doi:10.1016/j.leukres.2010.12.034

3. Warburg O. On the origin of cancer cells. Science. 1956;123 (3191):309-314. doi:10.1126/science.123.3191.309

4. Rajendran JG, Mankoff DA, O'Sullivan F, et al. Hypoxia and glucose metabolism in malignant tumors: evaluation by $[18 \mathrm{~F}]$ fluoromisonidazole and $[18 \mathrm{~F}]$ fluorodeoxyglucose positron emission tomography imaging. Clin Cancer Res. 2004;10(7):2245-2252. doi:10.1158/10780432.CCR-0688-3

5. Vander Heiden MG, Cantley LC, Thompson CB. Understanding the Warburg effect: the metabolic requirements of cell proliferation. Science. 2009;324(5930):1029-1033. doi:10.1126/science.1160809

6. Martinez-Outschoorn UE, Peiris-Pages M, Pestell RG, Sotgia F, Lisanti MP. Erratum: cancer metabolism: a therapeutic perspective. Nat Rev Clin Oncol. 2017;14(2):113. doi:10.1038/nrclinonc.2017.1
7. Zhang D, Tang Z, Huang H, et al. Metabolic regulation of gene expression by histone lactylation. Nature. 2019;574(7779):575-580. doi:10.1038/s41586-019-1678-1

8. Pajak B, Siwiak E, Soltyka M, et al. 2-Deoxy-d-glucose and its analogs: from diagnostic to therapeutic agents. Int $J$ Mol Sci. 2019;21(1):234. doi:10.3390/ijms21010234

9. Wang Q, Liang B, Shirwany NA, Zou MH. 2-Deoxy-D-glucose treatment of endothelial cells induces autophagy by reactive oxygen species-mediated activation of the AMP-activated protein kinase. PLoS One. 2011;6(2):e17234. doi:10.1371/journal.pone.0017234

10. Gerogianni I, Pitaraki E, Jagirdar RM, et al. 2-Deoxy-glucose enhances the effect of cisplatin and pemetrexed in reducing malignant pleural mesothelioma cell proliferation but not spheroid growth. Anticancer Res. 2019;39(7):3809-3814. doi:10.21873/anticanres.13530

11. Luo W, Semenza GL. Emerging roles of PKM2 in cell metabolism and cancer progression. Trends Endocrinol Metab. 2012;23 (11):560-566. doi:10.1016/j.tem.2012.06.010

12. Christofk HR, Vander Heiden MG, Harris MH, et al. The M2 splice isoform of pyruvate kinase is important for cancer metabolism and tumour growth. Nature. 2008;452(7184):230-233. doi:10.1038/ nature 06734

13. Amin S, Yang P, Li Z. Pyruvate kinase M2: a multifarious enzyme in non-canonical localization to promote cancer progression. Biochim Biophys Acta Rev Cancer. 2019;1871(2):331-341. doi:10.1016/j. bbcan.2019.02.003

14. Deng D, Xu C, Sun P, et al. Crystal structure of the human glucose transporter GLUT1. Nature. 2014;510(7503):121-125. doi:10.1038/ nature 13306

15. Cantor JR, Sabatini DM. Cancer cell metabolism: one hallmark, many faces. Cancer Discov. 2012;2(10):881-898. doi:10.1158/21598290.CD-12-0345

16. Kim I, Kwon D, Lee D, Lee G, Yoon DS. Permselective glucose sensing with GLUT1-rich cancer cell membranes. Biosens Bioelectron. 2019;135:82-87. doi:10.1016/j.bios.2019.04.007

17. Liu YX, Feng JY, Sun MM, et al. Aspirin inhibits the proliferation of hepatoma cells through controlling GLUT1-mediated glucose metabolism. Acta Pharmacol Sin. 2019;40(1):122-132. doi:10.1038/ s41401-018-0014-x

18. Tu Y. Artemisinin-A gift from traditional Chinese medicine to the world (Nobel lecture). Angew Chem Int Ed Engl. 2016;55 (35):10210-10226. doi:10.1002/anie.201601967

19. Wang J, Xu C, Liao FL, Jiang T, Krishna S, Tu Y. A temporizing solution to "artemisinin resistance". $N$ Engl J Med. 2019;380 (22):2087-2089. doi:10.1056/NEJMp1901233

20. Wong $\mathrm{YK}, \mathrm{Xu} \mathrm{C}$, Kalesh KA, et al. Artemisinin as an anticancer drug: recent advances in target profiling and mechanisms of action. Med Res Rev. 2017;37(6):1492-1517. doi:10.1002/med.21446

21. Huang L, Luo Y, Sun $\mathrm{X}$, Ju H, Tian J, Yu BY. An artemisinin-mediated ROS evolving and dual protease light-up nanocapsule for real-time imaging of lysosomal tumor cell death. Biosens Bioelectron. 2017;92:724-732. doi:10.1016/j.bios.2016.10.004

22. Lin R, Zhang Z, Chen L, et al. Dihydroartemisinin (DHA) induces ferroptosis and causes cell cycle arrest in head and neck carcinoma cells. Cancer Lett. 2016;381(1):165-175. doi:10.1016/j.canlet.2016.07.033

23. Chen X, Wong YK, Lim TK, et al. Artesunate activates the intrinsic apoptosis of HCT116 cells through the suppression of fatty acid synthesis and the NF-kB pathway. Molecules. 2017;22(8):1272. doi:10.3390/molecules 22081272

24. Chen SS, Hu W, Wang Z, Lou XE, Zhou HJ. p8 attenuates the apoptosis induced by dihydroartemisinin in cancer cells through promoting autophagy. Cancer Biol Ther. 2015;16(5):770-779. doi:10.1080/15384047.2015.1026477

25. Dong J, Chen Y, Yang W, Zhang X, Li L. Antitumor and anti-angiogenic effects of artemisinin on breast tumor xenografts in nude mice. Res Vet Sci. 2020;129:66-69. doi:10.1016/j. rvsc.2020.01.005 
26. Li Y, Zhou X, Liu J, Yuan X, He Q. Therapeutic potentials and mechanisms of artemisinin and its derivatives for tumorigenesis and metastasis. Anticancer Agents Med Chem. 2020;20. doi:10.2174/ 1871520620666200120100252

27. Li YH, Li XF, Liu JT, et al. PKM2, a potential target for regulating cancer. Gene. 2018;668:48-53. doi:10.1016/j.gene.2018.05.038

28. Palsson-McDermott EM, O’Neill LA. The Warburg effect then and now: from cancer to inflammatory diseases. Bioessays. 2013;35 (11):965-973. doi:10.1002/bies.201300084

29. Yeung SJ, Pan J, Lee MH. Roles of p53, MYC and HIF-1 in regulating glycolysis - the seventh hallmark of cancer. Cell Mol Life Sci. 2008;65(24):3981-3999. doi:10.1007/s00018-008-8224-x

30. Mathupala SP, Rempel A, Pedersen PL. Aberrant glycolytic metabolism of cancer cells: a remarkable coordination of genetic, transcriptional, post-translational, and mutational events that lead to a critical role for type II hexokinase. J Bioenerg Biomembr. 1997;29 (4):339-343. doi:10.1023/A:1022494613613

31. Sutendra G, Michelakis ED. Pyruvate dehydrogenase kinase as a novel therapeutic target in oncology. Front Oncol. 2013;3:1-11. doi:10.3389/fonc.2013.00038

32. Fantin VR, St-Pierre J, Leder P. Attenuation of LDH-A expression uncovers a link between glycolysis, mitochondrial physiology, and tumor maintenance. Cancer Cell. 2006;9(6):425-434. doi:10.1016/j. ccr.2006.04.023

33. Tamada M, Suematsu M, Saya H. Pyruvate kinase M2: multiple faces for conferring benefits on cancer cells. Clin Cancer Res. 2012;18 (20):5554-5561. doi:10.1158/1078-0432.CCR-12-0859

34. Mazurek S, Boschek CB, Hugo F, Eigenbrodt E. Pyruvate kinase type M2 and its role in tumor growth and spreading. Semin Cancer Biol. 2005;15(4):300-308. doi:10.1016/j.semcancer.2005.04.009
35. Anastasiou D, Poulogiannis G, Asara JM, et al. Inhibition of pyruvate kinase M2 by reactive oxygen species contributes to cellular antioxidant responses. Science. 2011;334(6060):1278-1283. doi:10.1126/ science. 1211485

36. Hsu MC, Hung WC. Pyruvate kinase M2 fuels multiple aspects of cancer cells: from cellular metabolism, transcriptional regulation to extracellular signaling. Mol Cancer. 2018;17(1):35. doi:10.1186/ s12943-018-0791-3

37. Zhang X, Chen B, Wu J, et al. Aspirin enhances the protection of Hsp90 from heat-stressed injury in cardiac microvascular endothelial cells through PI3K-Akt and PKM2 pathways. Cells. 2020;9(1):243. doi:10.3390/cells9010243

38. Li ZZ, Wang F, Liu S, Li H, Wang Y. Ablation of PKM2 ameliorated ER stress-induced apoptosis and associated inflammation response in IL-1beta-treated chondrocytes via blocking Rspo2-mediated Wnt/ beta-catenin signaling. J Cell Biochem. 2020.

39. Fox JM, Moynihan JR, Mott BT, et al. Artemisinin-derived dimer ART-838 potently inhibited human acute leukemias, persisted in vivo, and synergized with antileukemic drugs. Oncotarget. 2016;7(6):7268-7279. doi:10.18632/oncotarget.6896

40. Lai H, Singh NP. Selective cancer cell cytotoxicity from exposure to dihydroartemisinin and holotransferrin. Cancer Lett. 1995;91 (1):41-46. doi:10.1016/0304-3835(94)03716-V

41. Li S, Huang P, Gan J, et al. Dihydroartemisinin represses esophageal cancer glycolysis by down-regulating pyruvate kinase M2. Eur J Pharmacol. 2019;854:232-239. doi:10.1016/j.ejphar.2019.04.018

42. Grundker C, Wokoun U, Hellriegel M, Emons G. Inhibition of aerobic glycolysis enhances the anti-tumor efficacy of Zoptarelin Doxorubicin in triple-negative breast cancer cells. J Obstet Gynaecol Res. 2019;45(7):1334-1342. doi:10.1111/jog.13980

\section{Publish your work in this journal}

Drug Design, Development and Therapy is an international, peerreviewed open-access journal that spans the spectrum of drug design and development through to clinical applications. Clinical outcomes, patient safety, and programs for the development and effective, safe, and sustained use of medicines are a feature of the journal, which has also been accepted for indexing on PubMed Central. The manuscript management system is completely online and includes a very quick and fair peer-review system, which is all easy to use. Visit http://www. dovepress.com/testimonials.php to read real quotes from published authors. 